\title{
Perfil Antropométrico de Basquetbolistas Sub-14 Chilenos
}

\author{
Anthropometric Profile of Chilean Under-14 Basketball Players
}

Gajardo-Burgos, Rubén ${ }^{1,4}$; Barría Vargas, César ${ }^{2,4}$; Flández Valderrama, Jorge ${ }^{3,4}$; Avendaño Chipón, René ${ }^{4}$; Barría Pailaquilén, R. Mauricio ${ }^{1} \&$ Monrroy Uarac, Manuel $^{1,4}$

GAJARDO-BURgos, R.; BARRÍA, V. C.; FLÁNDEZ, V. J.; AVENDAÑO, C. R.; BARRÍA, P. R. M. \& MONRROY, U. M. Perfil antropométrico de basquetbolistas sub-14 chilenos. Int. J. Morphol., 36(3):943-947, 2018.

RESUMEN: La antropometría es un instrumento clave para determinar cambios producidos por el entrenamiento y la edad, así como una herramienta para detectar talentos deportivos. El objetivo de esta investigación fue describir las características antropométricas de basquetbolistas varones seleccionados chilenos sub-14 durante el año 2017. Se evaluaron 28 deportistas con una edad promedio de $14.14 \pm 0.36$ [13.33-14.63] los cuales participaron del proceso de concentración de la Selección Nacional. Se evaluaron 25 variables antropométricas. Se calculó la composición corporal pentacompartimental, el somatotipo y la proporcionalidad. Se utilizó estadística descriptiva para la caracterización de los deportistas. Se encontró que los basquetbolistas sub-14 chilenos presentan un porcentaje de masa muscular elevado, un somatotipo mesoectomórfico y proporciones (score Z) negativas respecto a su altura.

PALABRAS CLAVE: Antropometría, Somatotipo, Composición corporal, Básquetbol.

\section{INTRODUCCIÓN}

El básquetbol es uno de los deportes más practicados, estimándose 450 millones de jugadores según la Federación Internacional de Baloncesto. Este es un deporte de equipo que requiere de actividades intermitentes de alta intensidad, combinado con requerimientos técnicos específicos como avanzar corriendo con el balón, lanzar, saltar, pivotar, etc. (Ben Abdelkrim et al., 2007; Scanlan el al., 2011). Estas actividades requieren del desarrollo de distintas capacidades físicas para lograr un mejor rendimiento deportivo, entre ellas, la condición antropométrica (Torres-Unda et al., 2013).

El estudio antropométrico de los deportistas es un instrumento ampliamente utilizado para la caracterización funcional de los deportistas. Su utilización ha estado principalmente dirigido a valorar los cambios producidos por la maduración biológica del deportista (Gil et al., 2014a), el entrenamiento, la especialización deportiva y la competición (Hammami et al., 2013). También es de gran utilidad en la búsqueda de talentos deportivos (Gil et al., 2014b ; Gaudion et al., 2017), la mejora del rendimiento y la valoración de la condición de salud (Mountjoy et al., 2011). La actividad física en niños y jóvenes, particularmente las actividades que requieren aplicar fuerza rápidamente (saltos y carreras cortas, por ejemplo), genera cambios positivos en el tamaño y la estructura ósea del deportista (Gunter et al., 2012; Zribi et al., 2014) y, por otro lado, cambios en la composición corporal de los deportistas se asocian a modificaciones en sus capacidades físicas (Högström et al., 2012).

Muchos factores, entre ellos técnicos, tácticos, fisiológicos y antropométricos, están asociados a la identificación de jóvenes talentos (Strumbelj \& Erculj, 2014). En el basquetbol, las características antropométricas son determinantes en el rendimiento deportivo (Garcia-Gil et al., 2018), además de constituir uno de los principales factores para determinar la posición de juego del deportista; así en la posición de base se designan jugadores más bajos y de menor peso que en posiciones como las de pívot (Boone \& Bourgois, 2013).

A pesar de los beneficios de poseer evaluaciones objetivas de jóvenes deportistas considerados talentosos, a conocimiento de los autores, no existen datos de referencias antropométricas para generar un perfil útil para la detección

\footnotetext{
${ }^{1}$ Escuela de Kinesiología, Instituto de Aparato Locomotor y Rehabilitación, Universidad Austral de Chile, Valdivia, Chile.

${ }^{2}$ Federación de Basquetbol de Chile.

${ }^{3}$ Escuela de Pedagogía en Educación Física, Deporte y Recreación, Instituto de Filosofía y Estudios Educacionales, Universidad Austral de Chile, Chile.

${ }^{4}$ Programa de Entrenamiento Regional, Región de Los Ríos. Instituto Nacional del Deporte, Región de Los Ríos, Chile.
} 
de talentos y la monitorización de los cambios producidos por la maduración de estos deportistas y el efecto que tiene en ellos el entrenamiento y la competición. Es por esto que este estudio tuvo por objetivo caracterizar antropométricamente a los basquetbolistas seleccionados chilenos varones sub-14.

\section{MATERIAL Y MÉTODO}

En este estudio descriptivo transversal se evaluaron 28 jugadores de basquetbol varones menores de 14 años que participaron de los procesos de concentración de la Selección Nacional organizados por la Federación de Basquetbol de Chile durante los meses de julio y agosto del año 2017 en la ciudad de Valdivia. De este grupo de jugadores, 11 formaron parte de la Selección chilena U14 (sub-14) que participó en el Campeonato Sudamericano de Basquetbol U14.

Se midieron las variables utilizando el protocolo propuesto por la International Society for the Advancement of Kineanthropometry (ISAK) (Marfell-Jones et al., 2006). Las variables evaluadas fueron talla, peso, talla sentado y envergadura, diámetros biacromial, tórax transverso, tórax anteroposterior, biiliocrestídeo, humeral y femoral, perímetros de cabeza, brazo relajado, brazo en tensión, antebrazo máximo, tórax mesoesternal, cintura mínima, cadera máxima, muslo máximo, muslo medio y pantorrilla máxima, y los pliegues de tríceps, subescapular, supraespinal, abdominal, muslo medial y pantorrilla. Las medidas fueron tomadas por dos antropometristas, uno de ellos con acreditación ISAK nivel 2 y con una experiencia de 10 años. El registro inicial se realizó en un formulario impreso diseñado para este propósito y luego digitado en una planilla Excel para el cálculo de las variables.

Para la evaluación de las variables se utilizó un kit de antropometría Rosscraft S.R.L. de fabricación MERCOSUR, el cual posee un cáliper Gaucho Pro de 0,5 $\mathrm{mm}$ de precisión, antropómetro grande con $0,1 \mathrm{~mm}$ de precisión, antropómetro pequeño con $0,01 \mathrm{~mm}$ de precisión, una cinta métrica metálica de $1 \mathrm{~mm}$ de precisión y un segmómetro con $1 \mathrm{~mm}$ de precisión. Para la talla sentado se utilizó un cajón de madera de $40 \mathrm{~cm}$. Para la determinación del peso se utilizó una balanza MEDISANA PST modelo 40420 , la cual posee $100 \mathrm{~g}$. de precisión y una capacidad máxima de $180 \mathrm{~kg}$.

Los deportistas fueron evaluados con el mínimo de ropa posible. Antes de realizar la valoración de perímetros, diámetros y pliegues se realizaron marcas de referencias para disminuir el error de medición.
Para la determinación de la composición corporal se utilizó el modelo pentacompartimental descrito por Kerr (1988). Se realizó el cálculo de los valores $Z$ según el modelo de proporcionalidad del Phantom de Ross \& MarfellJones (1991). La superficie corporal (SC) se determinó utilizando la fórmula de Ross \& Kerr (1991): SC = Peso 0,425 * Talla $0,725 * 0.0068 .308$. Para el índice esquel se dividió la talla sentado por la talla corporal. Además, se caracterizó a los deportistas a través del somatotipo según el modelo de Carter \& Heath (1990), la sumatoria de 6 pliegues y la envergadura, que es la distancia entre los puntos dedales de la mano derecha y de la mano izquierda cuando las extremidades superiores están en abducción de $90^{\circ}$.

Se realizó un análisis estadístico descriptivo, utilizando como medida de tendencia central la media y de dispersión su desviación estándar, además de los valores mínimos y máximos. Se categorizó la muestra en seleccionados y no seleccionados para participar en el Campeonato Sudamericano de Basquetbol U14-2017, según criterios técnicos, y se comparó entre ellos las variables antropométricas utilizando test $\mathrm{T}$.

\section{RESULTADOS Y DISCUSIÓN}

El objetivo de este estudio fue caracterizar antropométricamente a los deportistas seleccionados chilenos varones sub-14. Para esto se realizó la caracterización y análisis de las variables antropométricas. Los valores de las medias y su respectiva desviación estándar, mínimo y máximo de las variables evaluadas se muestran en la Tabla I.

Los basquetbolistas sub-14 chilenos muestran un IMC de 21,77 $\pm 2,55$ lo cual los categoriza como normopeso. En un estudio realizado en Chile (Vargas et al., 2014), se evaluaron 4385 jóvenes de la misma edad de nuestra población, encontrándose un IMC similar $(22,09)$ a nuestros evaluados y mostrando claras diferencias en las variables de talla $(x=158,9)$ y peso $(x=55,93)$, en donde los seleccionados son más altos y de mayor peso, características esperadas en jugadores de basquetbol.

La composición corporal de la población de estudio muestra porcentajes de masa muscular de $41,82 \pm 2,82$, masa adiposa de $29,34 \pm 4,22$, masa ósea de $12,83 \pm 1,47$, masa residual de 10,39 $\pm 1,03$ y masa piel de 5,62 \pm 0,65 según el modelo pentacompartimental de Kerr. De estos, se destaca el elevado porcentaje de masa muscular, siendo esta valoración similar a otros estudios de basquetbolistas de la misma categoría, pero a diferencia de nuestro estudio ellos utiliza- 
Tabla I. Características de basquetbolistas sub-14 chilenos, Valdivia, Chile.

\begin{tabular}{lcccc}
\hline & Media & DE & Mínima & Máxima \\
\hline Edad (años) & 14,14 & 0,36 & 13,3 & 14,6 \\
Peso (kg) & 73,54 & 12,81 & 51,1 & 106 \\
Talla (cm) & 183,24 & 7,62 & 164,3 & 198,7 \\
IMC (kg/m ${ }^{2}$ ) & 21,77 & 2,55 & 17,9 & 28,8 \\
Talla sentado (cm) & 93,96 & 4,53 & 82,8 & 105,4 \\
Envergadura (cm) & 186,34 & 9,61 & 169,5 & 205,0 \\
PL Tríceps (mm) & 10,54 & 4,27 & 5,5 & 20,0 \\
PL Subescapular (mm) & 8,95 & 4,22 & 5,0 & 20,5 \\
PL Supraespinal (mm) & 11,79 & 7,90 & 3,5 & 36,0 \\
PL Abdominal (mm) & 19,04 & 11,61 & 4,5 & 49,0 \\
PL Muslo frontal (mm) & 12,64 & 3,76 & 7,0 & 22,0 \\
PL Pantorrilla medial (mm) & 9,71 & 4,03 & 5,0 & 20,0 \\
Suma de 6 pliegues & 72,67 & 33,34 & 34,0 & 162,5 \\
PR Brazo relajado (cm) & 27,75 & 2,73 & 24,0 & 34,4 \\
PR Brazo flexionado en tensión (cm) & 28,70 & 2,29 & 25,1 & 33,4 \\
PR Antebrazo máximo (cm) & 25,56 & 1,42 & 22,5 & 28,8 \\
PR Tórax mesoesternal (cm) & 90,16 & 6,98 & 77,4 & 107,0 \\
PR Cintura (cm) & 77,08 & 8,09 & 65,7 & 95,4 \\
PR Cadera (cm) & 94,90 & 7,10 & 79,5 & 110,8 \\
PR Muslo (cm) & 55,61 & 4,96 & 46,6 & 67,4 \\
PR Muslo medial (cm) & 50,22 & 4,09 & 44,0 & 59,3 \\
PR Pantorilla (cm) & 36,75 & 2,51 & 32,5 & 42,9 \\
D Biacromial (cm) & 39,86 & 2,10 & 34,7 & 43,9 \\
D Tórax transverso (cm) & 27,36 & 1,93 & 23,2 & 31,7 \\
D Tórax antero-posterior (cm) & 18,73 & 1,50 & 16,1 & 22,2 \\
D Bi-iliocrestídeo (cm) & 29,21 & 2,65 & 23,7 & 34 \\
D Humeral (cm) & 7,46 & 0,35 & 6,9 & 8,45 \\
D Femoral (cm) & 10,58 & 0,43 & 9,8 & 11,6 \\
\hline
\end{tabular}

PL: pliegues; PR: perímetros; D: diámetros. ron métodos tricompartimentales (TorresUnda et al.; Campo et al., 2016). A nuestro conocimiento, este es el primer estudio bajo este modelo de basquetbolistas sub-14. La valoración y seguimiento de los cambios de la composición corporal en deportistas es de gran importancia ya que cambios en esta, como un aumento de la masa muscular y disminución de la masa adiposa, se asocian a mejoras en el rendimiento aeróbico y en acciones tan importantes en el basquetbol como lo es el salto (Högström et al.; Santos et al., 2014).

La proporcionalidad establece la relación de las distintas partes del cuerpo en base a proporciones ideales. Esta es de gran importancia debido a que los deportistas de alto rendimiento tienden a tener proporciones similares (Almagiá et al., 2015). Respecto a los sujetos evaluados, éstos presentaron principalmente valores negativos en sus variables, a excepción de los diámetros humerales y femorales. Este es un resultado esperable ya que los valores se ajustan a la variable talla y es esta la principal característica de los basquetbolistas (Fig. 1).

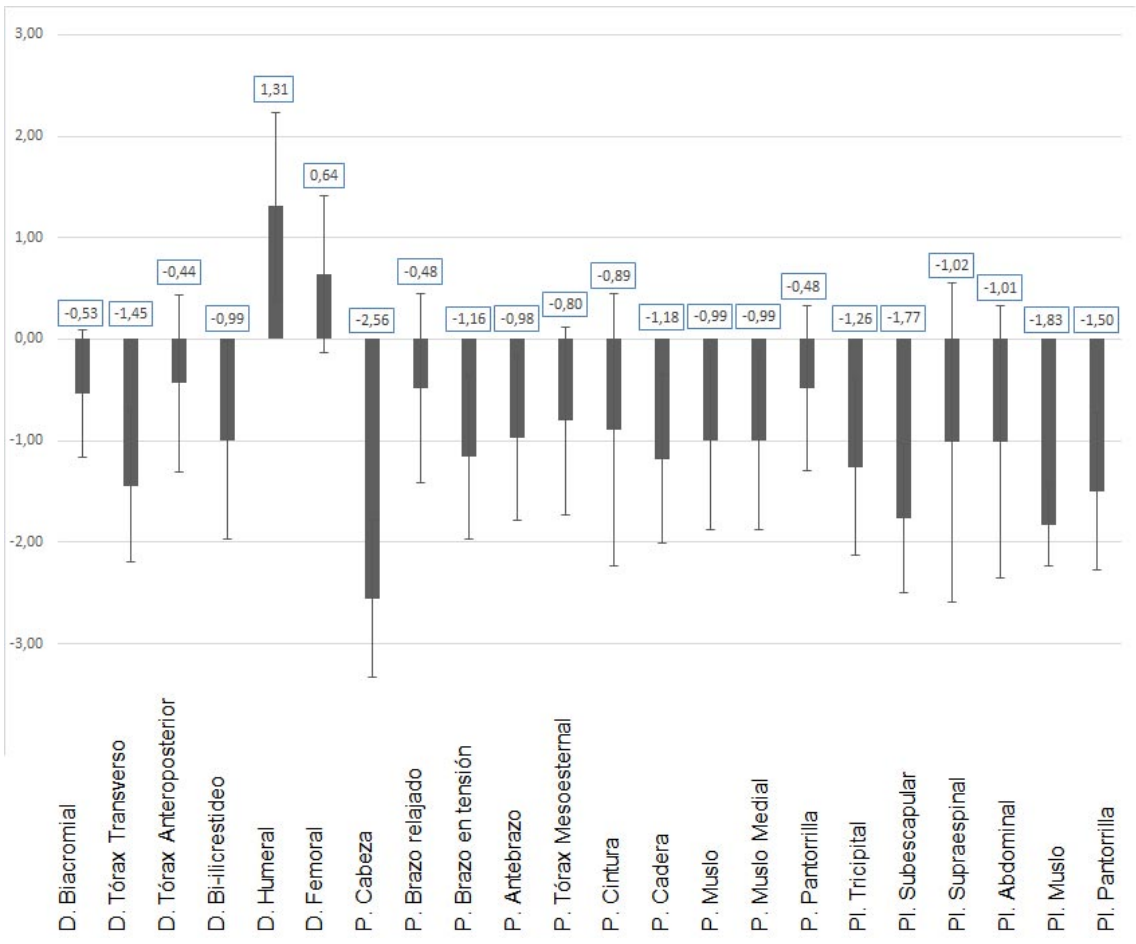

Fig. 1. Proporcionalidad (valores Z) de basquetbolistas sub-14 chilenos. En el cuadro valor promedio. D: diámetro; P: perímetro; Pl: pliegue. 
El somatotipo establece diferencias en la forma corporal y permite conocer el estado físico y nutricional de una población deportiva, así como comparar diferentes individuos y especialidades, pudiendo mostrar tendencias antropométricas. Nuestra población se categorizó como mesoectomórfico $(2,85-4,20-3,58)$ lo cual indica un alto desarrollo musculoesquelético, poco tejido adiposo subcutáneo y una elevada altura (Norton \& Olds, 1996). Esta categorización muestra diferencias con la población general del mismo rango etario (Martínez et al., 2008), ya que los basquetbolistas muestran una ma- yor característica ectomórfica (3,6 vs 2,4), lo cual está relacionado con su mayor altura, condición común en este tipo de población (Campo et al.; Rivera-Sosa, 2016) (Fig. 2).

Finalmente, la comparación de los deportistas seleccionados nacionales para el Campeonato Sudamericano de Basquetbol U14 mostró una tendencia a que estos son más altos, con mayor envergadura, mas ectomórficos y con mayor masa muscular, versus los no seleccionados, tal como se muestra en la Tabla II.

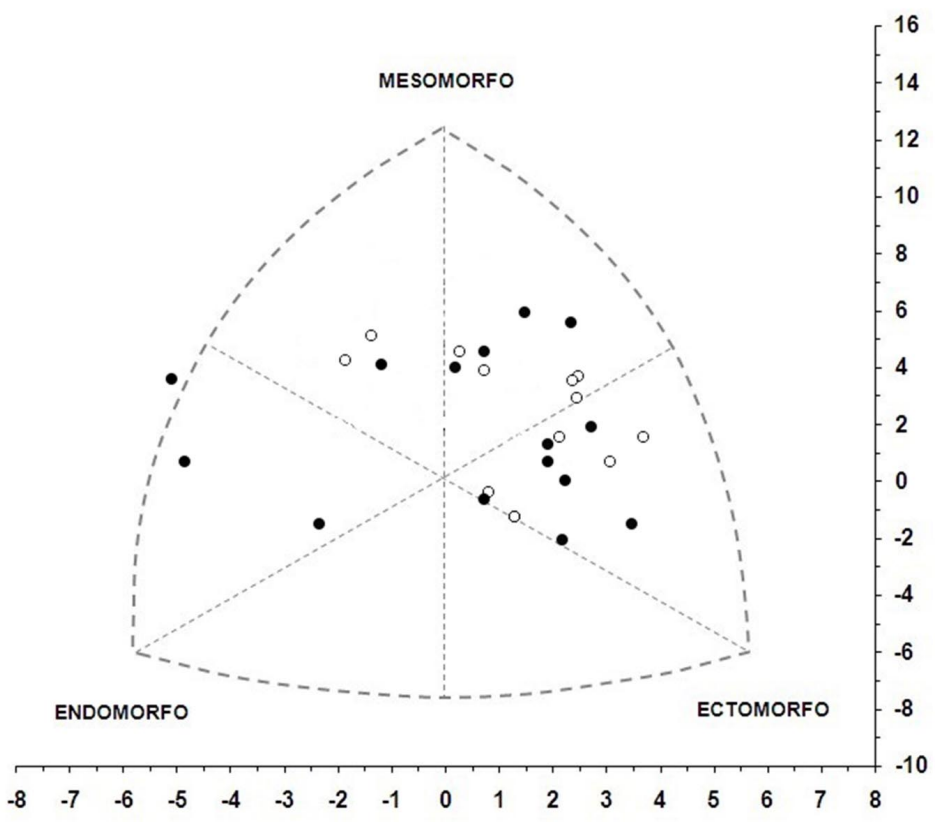

Fig. 2. Somatocarta de basquetbolistas sub-14 chilenos. Los círculos negros pertenecen a basquetbolistas pre-seleccionados. Los círculos blancos pertenecen a basquetbolistas participantes del Campeonato Sudamericano de Basquetbol U14-2017.

\section{CONCLUSIÓN}

Los basquetbolistas chilenos sub-14 mostraron un porcentaje de masa muscular elevado, de tejido adiposo bajo y un somatotipo mesoectomórfico. Los seleccionados versus los no seleccionados tuvieron una tendencia a tener mejores resultados en las variables de altura, envergadura, masa muscular y masa adiposa. Este artículo aporta una descripción antropométrica de los basquetbolistas seleccionados chilenos sub-14, pudiendo ser utilizados para futuros procesos de búsquedas de talentos, análisis de los cambios morfológicos que pueden generar planes de entrenamientos, nutrición y la maduración.

Tabla II. Comparación basquetbolistas sub-14 chilenos seleccionados versus no seleccionados, Valdivia, Chile.

\begin{tabular}{lccc}
\hline \multicolumn{1}{c}{ Variables } & $\begin{array}{c}\text { Seleccionados } \\
(11)\end{array}$ & $\begin{array}{c}\text { No Seleccionados } \\
(17)\end{array}$ & Valor $p$ \\
\hline Peso $(\mathrm{kg})$ & $75,99 \pm 11,33$ & $71,95 \pm 13,78$ & 0,4250 \\
Talla (cm) & $186,37 \pm 6,15$ & $181,21 \pm 7,96$ & 0,0802 \\
Envergadura $(\mathrm{cm})$ & $189,39 \pm 9,04$ & $184,37 \pm 9,70$ & 0,1816 \\
Endomorfismo & $2,47 \pm 0,95$ & $3,09 \pm 1,59$ & 0,2582 \\
Mesomorfimo & $4,21 \pm 0,93$ & $4,19 \pm 1,02$ & 0,9689 \\
Ectomorfismo & $3,73 \pm 0,92$ & $3,47 \pm 1,12$ & 0,5316 \\
Masa adiposa $(\%)$ & $27,91 \pm 3,28$ & $30,26 \pm 4,59$ & 0,1554 \\
Masa muscular $(\%)$ & $42,95 \pm 2,37$ & $41,09 \pm 2,91$ & 0,0895 \\
Masa ósea $(\%)$ & $13,07 \pm 1,36$ & $12,68 \pm 1,56$ & 0,4974 \\
Masa residual $(\%)$ & $10,42 \pm 0,81$ & $10,37 \pm 1,18$ & 0,9185 \\
Masa piel $(\%)$ & $5,66 \pm 0,49$ & $5,60 \pm 0,75$ & 0,8427 \\
\hline
\end{tabular}


GAJARDO-BURGOS, R.; BARRÍA, V. C.; FLÁNDEZ, V. J.; AVENDAÑO, C. R.; BARRÍA, P. R. M. \& MONRROY, U. M. Anthropometric profile of Chilean under-14 basketball players. Int. J. Morphol., 36(3):943-947, 2018.

SUMMARY: Anthropometry is a key instrument to find body changes produced by sports training and age. It is also being used to determine young skilled athletes. The aim of this study was to determine the anthropometric characteristics of Chilean male basketball players under 14 years of age during 2017. We measured 28 athletes with an average age of $14.14 \pm 0.36$ [13.3314.63] who participated in the National Chilean Team concentration process. In the study, 25 anthropometric variables were measured including penta compartimental body composition, somatotype and proportionality were estimated. Statistical description was applied for the athletes characterization. The analysis showed that Chilean U-14 basketball players have a high percentage of muscle mass, a meso-ectomorphic somatotype and negative proportions ( $\mathrm{Z}$ score) regarding their height.

KEY WORDS: Anthropometry; Somatotype; Body composition; Basketball.

\section{REFERENCIAS BIBLIOGRÁFICAS}

Almagiá, A.; Araneda, A.; Sánchez, J.; Sánchez, P.; Zuñiga, M. \& Plaza, P. Elección del modelo de proporcionalidad antropométrica en una población deportista; comparación de tres métodos. Nutr. Hosp., 32(3):1228-33, 2015.

Ben Abdelkrim, N.; El Fazaa, S. \& El Ati, J. Time-motion analysis and physiological data of elite under-19-year-old basketball players during competition. $\mathrm{Br}$. J. Sports Med., 41(2):69-75, 2007.

Boone, J. \& Bourgois, J. Morphological and physiological profile of elite basketball players in Belgian. Int. J. Sports Physiol. Perform., 8(6):630-8, 2013.

Campo, M. A.; Escortell Sánchez, R.; Sospedra, I.; Norte-Navarro, A.; MartínezRodríguez, A. \& Martínez-Sanz, J. M. Características cineantropométricas en jugadores de baloncesto adolescentes. Rev. Esp. Nutr. Hum. Diet., 20(1):23$31,2016$.

Carter, J. E. L. \& Heath, B. H. Somatotyping: Development and Application. Cambridge, Cambridge University $\mathrm{P}$ ess, 1990.

Garcia-Gil, M.; Torres-Unda, J.; Esain, I.; Duñabeitia, I.; Gil, S. M.; Gil, J. \& Irazusta, J. Anthropometric parameters, age, and agility as performance predictors in elite female basketball players. J. Strength Cond. Res., 32(6):172330, 2018.

Gaudion, S. L.; Doma, K.; Sinclair, W.; Banyard, H. G. \& Woods, C. T. Identifying the physical fitness, anthropometric and athletic movement qualities discriminant of developmental level in elite junior australian football: Implications for the development of talent. J. Strength Cond. Res., 31(7):18309, 2017

Gil, S. M.; Badiola, A.; Bidaurrazaga-Letona, I.; Zabala-Lili, J.; Gravina, L.; Santos-Concejero, J.; Lekue, J. A. \& Granados, C. Relationship between the relative age effect and anthropometry, maturity and performance in young soccer players. J. Sports Sci., 32(5):479-86, 2014a.

Gil, S. M.; Zabala-Lili, J.; Bidaurrazaga-Letona, I.; Aduna, B.; Lekue, J. A.; Santos-Concejero, J. \& Granados, C. Talent identification and selection process of outfield players and goalkeepers in a professional soccer club. J. Sports Sci., 32(20):1931-9, 2014b.

Gunter, K. B.; Almstedt, H. C. \& Janz, K. F. Physical activity in childhood may be the key to optimizing lifespan skeletal health. Exerc. Sport Sci. Rev., 40(1):13$21,2012$.

Hammami, M. A.; Ben Abderrahmane, A.; Nebigh, A.; Le Moal, E.; Ben Ounis, O.; Tabka, Z. \& Zouhal, H. Effects of a soccer season on anthropometric characteristics and physical fitness in elite young soccer players. J. Sports Sci., 31(6):589-96, 2013.

Högström, G. M.; Pietilä, T.; Nordström, P. \& Nordström, A. Body composition and performance: influence of sport and gender among adolescents. J. Strength Cond. Res., 26(7):1799-804, 2012.

Kerr, D. A. An Anthropometric Method for Fractionation of Skin, Adipose, Bone, Muscle and Residual Masses in Males and Females Age 6 to 77 Years. Master Kinesiology Tesis, British Columbia, Simon Fraser University, 1988.

Marfell-Jones, M.; Olds, T.; Stewart, A. \& Carter, L. International Standards for Anthropometric Assessment. Potchefstroom, ISAK, 2006.

Martínez, C.; Silva, H.; Collipal, E. \& Carrasco, S. V. Description of somatotype and BMI the adolescent's sample to public school of the Temuco - Chile. Int. J. Morphol., 26(3):653-7, 2008.

Mountjoy, M.; Andersen, L. B.; Armstrong, N.; Biddle, S.; Boreham, C.; Bedenbeck, H. P.; Ekelund, U.; Engebretsen, L.; Hardman, K.; Hills, A. P.; Kahlmeier, S.; Kriemler, S.; Lambert, E.; Ljungqvist, A.; Matsudo, V.; McKay, H.; Micheli, L.; Pate, R.; Riddoch, C.; Schamasch, P.; Sundberg, C. J.; Tomkinson, G.; van Sluijs, E. \& van Mechelen, W. International Olympic Committee consensus statement on the health and fitness of young people through physical activity and sport. Br. J. Sports Med., 45(11):839-48, 2011.

Norton, K. \& Olds, T. Antropométrica. Marrickville, Southwood Press, 1996.

Rivera-Sosa, J. M. Anthropometric properties and somatotype of basketball players from different competition level. Int. J. Morphol., 34(1):179-88, 2016.

Ross, W. D. \& Kerr, D. A. Fraccionamiento de la masa corporal: um nuevo metodo para utilizar en Nutricion Clinica y Medicina Deportiva. Rev. Apunt. Med. Deport., 18:175-87, 1991.

Ross, W. D. \& Marfell-Jones, M. Kinanthropometry. En: MacDougal, J.; Wenger, H. \& Green, H. (Eds.). Physiological Testing of the High Performance Athlete. $2^{\text {nd }}$ ed. Champaign (IL), Human Kinetics, 1991. pp.223-308.

Santos, D. A.; Matias, C. N.; Rocha, P. M.; Minderico, C. S.; Allison, D. B.; Sardinha, L. B. \& Silva, A. M. Association of basketball season with body composition in elite junior players. J. Sports Med. Phys. Fitness, 54(2):16273, 2014.

Scanlan, A.; Dascombe, B. \& Reaburn, P. A comparison of the activity demands of elite and sub-elite Australian men's basketball competition. J. Sports Sci., 29(11):1153-60, 2011.

$\mathrm{S}$ trumbelj, E. \& Erculj, F. Analysis of experts' quantitative assessment of adolescent basketball players and the role of anthropometric and physiological attributes. J. Hum. Kinet., 42:267-76, 2014.

Torres-Unda, J.; Zarrazquin, I.; Gil, J.; Ruiz, F.; Irazusta, A.; Kortajarena, M.; Seco, J. \& Irazusta, J. Anthropometric, physiological and maturational characteristics in selected slite and non-elite male adolescent basketball players. J. Sports Sci., 31(2):196-203, 2013.

Vargas, V. R.; Bahamonde, P. C.; Cancino, L. J.; Correa, E. P.; Michelow, B. S.; Gatica, M. P.; Gómez, C. R.; Martínez, S. C.; Vargas, V. D.; Luna S. D. \& Cossio-Bolaños, M. Parámetros de crecimiento y adiposidad corporal de adolescentes chilenos a través de la referencia CDC-2000 y CDC-2012: Estudio ACECH. Rev. Chil. Nutr., 41(1):54-60, 2014.

Zribi, A.; Zouch, M.; Chaari, H.; Bouajina, E.; Zaouali, M.; Nebigh, A. \& Tabka, $\mathrm{Z}$. Enhanced bone mass and physical fitness in prepubescent basketball players. J. Clin. Densitom., 17(1):156-62, 2014.

\section{Dirección para correspondencia:}

Klgo. Mg. Rubén Gajardo Burgos

Profesor Auxiliar

Klgo. Manuel Monrry Uarac

Profesor Instructor

Escuela de Kinesiología

Universidad Austral de Chile

Rudloff 1650

Valdivia - CHILE

Recibido : 28-01-2018

Aceptado: 14-04-2018

Email: manuelmonrroy@uach.cl 